International Journal of Distributed and Parallel Systems (IJDPS) Vol.2, No.2, March 2011

\title{
A COMPARISON STUDY FOR THE BER IN BROADBAND FIXED WIRELESS ACCESS SYSTEMS BASED ON USING M-QAM
}

\author{
Haider M. AlSabbagh ${ }^{1}$ and Ali Amin ${ }^{2}$ \\ Department of Electrical Engineering, College of Engineering, Basra University \\ Basra - Iraq \\ ${ }^{1}$ haidermawieee.org $\quad 2$ ali_amindieee.org
}

\begin{abstract}
An analytical model for the bit error rate with different values of M-ary quadrature amplitude modulation $(M-Q A M)$ for broadband fixed wireless access (BFWA) is presented. The expressions/relations are derived and investigated for different values of $M: 4,8,16,32,64,128$, and 256 to evaluate conditional probability of error, bit error probability and average of the probability of error. The analyses are presented for coherent and non-coherent detection schemes and comparisons between their performances are given. The achieved results show that with keeping a fixed amount of symbols energy for all considered levels/symbols the degradation in BER is remarkable with low SNR with using non-coherent detection compared with utilizing coherent one for all M-ary values. Also, less power is needed in order to achieve the same BER with utilizing non-coherent detection method.
\end{abstract}

\section{KEYWORDS}

BER, Broadband, BFWA, QAM, M-QAM

\section{INTRODUCTION}

Recent years an exponential growth in numerous applications within the Internet as a whole seem the main interesting point to move towards support searching an efficient way to convey different types of data. Transmissions of such data not only require integrating the different kind of them, such that combine voice, video (e.g. TV, teleconference) and data services over a common network but efficient delivery of such a huge traffic with such applications requires broadband coverage networks. These are not currently available in almost all regions within many countries such as the European Union (EU) due to geography (e.g. mountains), small rural communities, or peripheral regions, creating a "digital divide" [1]. Thus, availability of the services become far from the universal and contention will seriously degrade throughput per user, and will necessitate the use of broadband wireless technology to integrate these users within the global internet $[1,2]$. Recently, using broadband fixed wireless access (BFWA) has several advantages to cope with the problem. It enables operators in a competitive environment to roll-out wideband services in a rapid and cost efficient manner [3, 4]. The IEEE working group on broadband wireless access standards developed IEEE 802.16 [5] that provides the standards for broadband wireless system implementation. OFDM has been proposed as a part of the specification in order to combat frequency-selective fading in the BFWA channel. Also, the use of space diversity for capacity improvement using OFDM has been analyzed in [6]. It is based on using quadrature amplitude modulation (QAM) which is a technique aimed to increase the spectral efficiency in communication systems. It is a modulation format for realizing optical [7-9] and wireless [10,11] communication systems with a high spectral efficiency of much greater than $1 \mathrm{bit} / \mathrm{s} / \mathrm{Hz}$. Compared with OOK (On-off-keying), 2M QAM signal processes M 
single channel which it has M times superiors in spectral efficiency. The QAM signal processes $M$ bits in a single channel, so it can realize $M$ times spectral efficiency compared with OOK. However, using QAM means the transmitted energy per period of the spreading sequence would vary and depending upon the particular QAM symbol being transmitted. Therefore, each channel would experience a varying amount of interference depending upon the particular combination of symbols being transmitted by the other channels [12].

The bit-error rate (BER) is of fundamental interest in digital communications [13], since the recovered signals depends upon the number of bits transmitted per second and bit error rate. Szczecinski et. al, [14] developed a closed-form formula for the uncoded BER of rectangular QAM modulation using non-Gray mapping. They consider it for coherent detection with two different of modulation levels, specifically, 16-QAM and 64-QAM. Researchers in [15] have discussed high peak-to-mean envelope power ratio which is the major issue in the implementation of orthogonal frequency division multiplexing systems in low application devices by introducing a star 16QAM (S16QAM). However, neither higher orders nor influence of different orders on bit error rate are discussed. A solution has been proposed in [16] with utilising QAM constellation, called A16QAM, to overcome problems with S16QAM in terms of average of symbol error rate (SER) and the constellation figure of merit (CFM), nevertheless, the analyses presented for $M=16$. On the other hand, Khan et. al., [17] show that overcoming the inter-symbol interference (ISI) effects of the channel equalization techniques are employed with low density parity check (LDPC) codes. The results were investigated and compared for systems employing QPSK and 16-QAM modulation schemes. However, influence of the other levels of the M-ary on the amount of the BER is not considered. In [3] the results were presented theoretically for BFWA system using coherent and non-coherent detection methods for QPSK and 16-QAM with equal value of the average symbol energy. In this paper, an analytical model for the bit error rate with different $\mathrm{M}$-ary of quadrature amplitude modulation (M-QAM) is presented. The analysis includes coherent and non-coherent detection methods. Then, comparisons for the BER with different $\mathrm{M}$ of QAM for the signal to noise ratio are studied. The achieved results show that with keeping fixed amount of symbols energy for all considered values/symbols the degradation in BER is remarkably noted with adopting coherent detection than that with employing non-coherent one. On the other side, keeping only fixed value of the encoded symbol leads to that amount of degradation with adopting non-coherent detection is greater than that with coherent one, while an improvement might be achieved with using lower values of M-ary. This paper is outlined as follows. Section 2 gives an analysis for the M-QAM for the two methods of detection, coherent and non-coherent. The performance analyses with the achieved results are presented in Section 3. The derived analytical expressions for the conditional bit error probability, error probability and average of probability of errors are listed for coherent and non-coherent detection methods in appendices A and B, respectively. The conclusions are drawn in Section 4.

\section{ANALYSIS OF M-QAM}

In quadrature amplitude modulation (QAM) the carrier signal is split into two amplitudes which are modulated independently with the same frequency; shifted in phase by 90 degrees. These carries are called in-phase carriers (I) and quadrature-phase carriers (Q). The QAM can assign $2^{\mathrm{k}}$-state by using I and Q, which is called $2^{\mathrm{k}}-\mathrm{QAM}$ (or M-QAM).

Figure 1 illustrates constellation maps for a $2^{\mathrm{k}}, \mathrm{k}=1,2, \ldots . .8$ which will be considered in the analyses. It is plotted for different types (a to y) to represent constellation of the different used values of $\mathrm{M}$ (4-256) in this paper. A discrete-time L-tap transversal filter is employed to model the multipath utilized channel. The impulse response: $h_{n}$ is given as: $\sum_{i=0}^{l-1} h_{i} \delta_{n-1}$ where $\mathrm{h}_{\mathrm{i}}$ is the complex channel coefficient.

Assuming the received signal from the channel is given by: 


$$
r_{n}=h_{o} s_{n}+w_{n}
$$

Each of $h_{0}, s_{n}$, and $w_{n}$ consists of two components; in-phase: $h_{I}, A, w_{n I}$ and quadrature component: $h_{Q}, A, w_{n Q}$, respectively, where $w_{n}$ is the term which takes into account influence

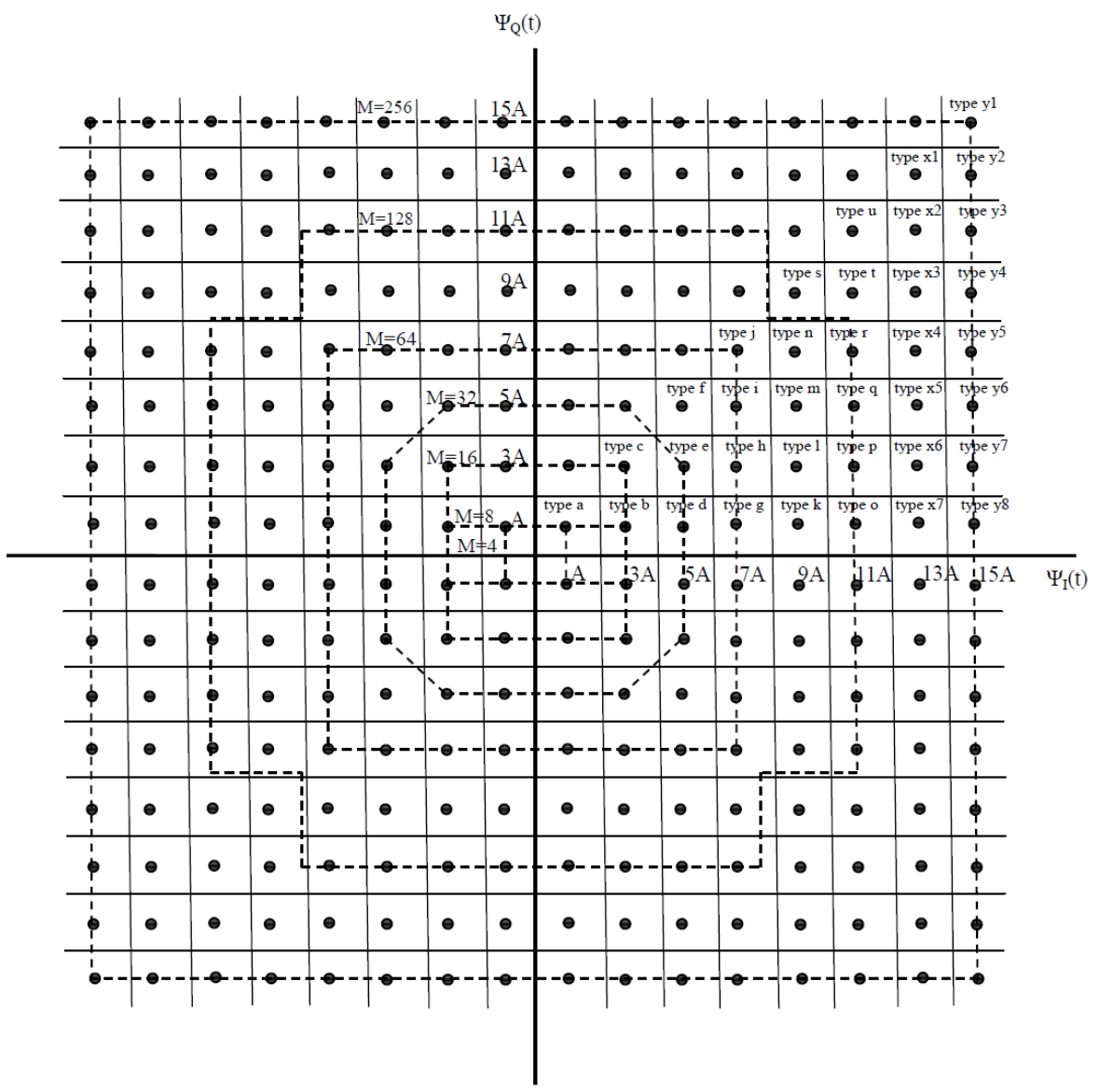

Figure 1. Space diagrams of several signals for rectangular QAM.

of ISI $\left(h_{1} s_{n-1}+h_{2} s_{n-2}\right)$ and the noise $\left(v_{n}\right)$, and the variance of the additive Gaussian noise is: $\mathrm{N}_{\mathrm{n}}=\mathrm{E}\left[\left|\mathrm{h}_{1}\right|^{2}\right]+\mathrm{E}\left[\left|\mathrm{h}_{2}\right|^{2}\right]+\mathrm{N}_{\mathrm{O}}=\mathrm{P}_{1}+\mathrm{P}_{2}+\mathrm{N}_{\mathrm{O}} ; \mathrm{w}_{\mathrm{n}}\left(=\mathrm{w}_{\mathrm{nI}}+\mathrm{jw} \mathrm{w}_{\mathrm{nQ}}\right)$ has a complex Gaussian distribution. In general, the probability of 1 and 2 errors events are given as in Ref. [3] and some of parameters values are used here to complete the derivation and facilitate process of comparison and checking.

\section{A- Non-coherent detection}

Before presenting the derivations, let us define some parameters to assist arranging the required relations/equations.

Let, $\quad Z_{1}=\frac{\sqrt{2} A\left(h_{I}-h_{Q}\right)}{\sqrt{N_{w}}} \quad, \quad Z_{2}=\frac{\sqrt{2} A\left(h_{I}+h_{Q}\right)}{\sqrt{N_{w}}}$

And, $\quad \gamma_{a}=n_{a 1 I} \cdot P_{1 I}+n_{a 1 Q} \cdot P_{1 Q}+n_{a 2} \cdot 2 \cdot P_{2}$

$$
\begin{aligned}
& \gamma_{b}=n_{b 1 I} \cdot P_{1 I}+n_{b 1 Q} \cdot P_{1 Q}+n_{b 2} \cdot 2 \cdot P_{2} \\
& \gamma_{c}=n_{c 1 I} \cdot P_{1 I}+n_{c 1 Q} \cdot P_{1 Q}+n_{c 2} \cdot 2 \cdot P_{2}
\end{aligned}
$$


And, $\psi=\frac{\sqrt{2} A \cdot m_{1}}{\sqrt{N_{w}+2 \cdot A^{2} \cdot \sigma}}$, where, $m_{1}$ is the mean of $h_{I}-h_{Q}$.

The probability of 1 error along the $\mathrm{I}$ axis is denoted as $P_{1 I}=Q\left(z_{1}\right)$ and the probability of 1 error along the quadrature component is: $P_{1 Q}=Q\left(Z_{2}\right)$, where $Q(z)=\int_{z}^{\infty} \frac{1}{\sqrt{2 \pi}} \mathrm{e}^{-x^{2} / 2} d x$ is the complementary Gaussian cumulative distribution function, $Q(0)=\frac{1}{2}$. While probability of occurring two decoded errors is given as: $P_{2}=Q\left(z_{1}\right) \cdot Q\left(z_{2}\right)$. The derivation for the probabilities of conditional bits and for the bit error rates and averaging of the error with employing non-coherent detection methods is given in appendix A.

\section{B- Coherent detection}

QAM is the standard coherent modulation scheme [10]. It is a popular choice because it uses bandwidth efficiently and linear channel distortions can be corrected by adaptive equalization at the receiver. QAM can be considered a logical extension of QPSK, since QAM also consists of two independently amplitude-modulated carriers in quadrature. Each block of $\mathrm{k}$ bits can be split into two (k/2)-bit blocks which use (k/2)-bit digital-to-analog (D/A) converters to provides the required modulating voltages for the carriers. At the receiver, each of the two signals is independently detected using matched filters [13].

Let, $v=2 \cdot \sqrt{2 \pi} \sigma^{3} \cdot \exp \left(\frac{\left(\frac{s}{\sigma}\right)^{2}}{2}\right) \cdot \sum_{n=1}^{\infty} \frac{s^{2 n-2}(2 n-1) ! !}{\left(2 \sigma^{2}\right)^{2 n}[(n-1) !]^{2}}$, where $n$ is an integer takes $0,1,2$, $\ldots$ and $(2 n-1) ! !=1.3 .5 \ldots(2 n-1)$. The average of the error probability can be derived to be as the following form, regardless value of the used M-ary:

$\bar{P}_{\text {ber }}=\frac{2}{3} P_{\text {ber }}(v)+\frac{1}{6} \cdot P_{\text {ber }}(v+\sqrt{3} \beta)+\frac{1}{6} \cdot P_{\text {ber }}(v-\sqrt{3} \beta)$,

where, $\beta=\sqrt{E\left[\left|h_{o}\right|^{2}\right]-v^{2}}$, and $P_{b e r}$ is the bit error probability, see appendix (B).

\section{Performance Analysis:}

To evaluate influence of signal to noise ratio (SNR) and energy of the transmitted signal on the overall performance of the system two cases will be considered: the transmitted symbols are normalized to unit energy $(=1)$ and for varying energy for each transmitted symbol (different with different values of the M-ary). For this end an expression for the average bit error probability $\left(\bar{P}_{e}\right)$ with the SNR are derived for the two detection schemes using with BFWA, namely, coherent and non-coherent methods. The derived expressions/relations presented in the previous section and listed in appendices A and B are employed in the evaluation of the system performance.

The derived expressions for average BER's for M-QAM are computed numerically and results are plotted in Fig. $2-\mathrm{a}$ and $-\mathrm{b}$ and Fig. $3-\mathrm{a}$ and $-\mathrm{b}$ for normalized and variable energy for the transmitted signals, respectively, each for: (a) coherent and (b) non-coherent schemes. The computations are presented with using the values given in Ref. [3] as: variance of $h_{0}, h_{1}$ and $h_{2}$ are 0.5, 0.3162 and 0.1 , respectively, mean of $h_{1}=h_{2}=0$, mean of $h_{0}=\sqrt{0.5}(1+j)$. The results are plotted for various values of $\mathrm{k}$ (number of bits per each symbol) to represent of the level $(\mathrm{M})$ in the M-QAM $\left(2^{2}, 2^{3}, 2^{4}, 2^{5}, 2^{6}, 2^{7}\right.$, and $\left.2^{8}\right)$ to examine the dependency of BER on parameter $\mathrm{M}$ (and hence $\mathrm{k}$ ). 


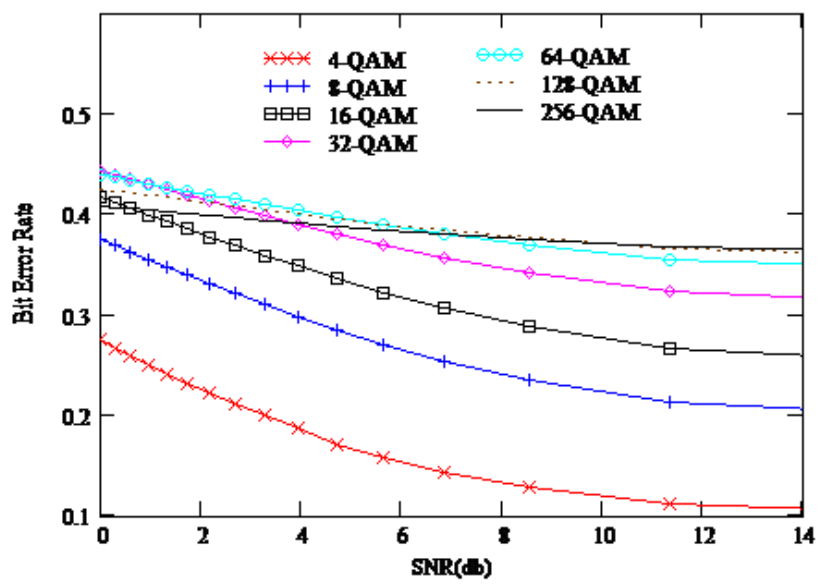

(a)

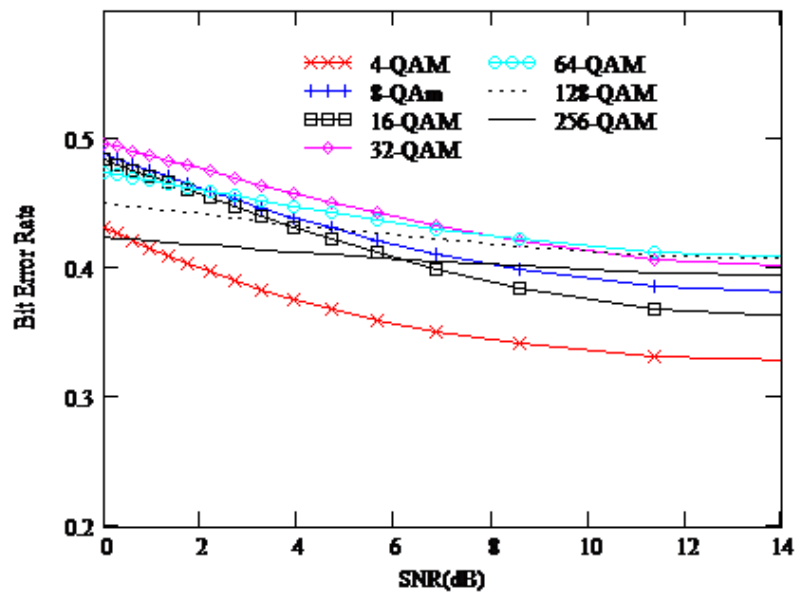

(b)

Figure 2. Bit error rate as a function of SNR for multi levels of QAM: 4, 8, 16, 32, 64, 128, and 256 over the BFWA channel for: (a) coherent and (b) non-coherent detection. (normalized energy is constant with all levels, Es=1).

With adopting coherent detection the degradation in the BER is obvious with $\mathrm{M}$ due to increasing probability of error, see appendix A. At low level of SNR $(<4 \mathrm{~dB})$ the BER with $\mathrm{k}>$ 3 have approximately equal values. And, BERs get an improvement with increasing SNR (> $6 \mathrm{~dB}$ ), high level of QAM get low change for the better and becomes almost no progress for $\mathrm{k}>$ 5 while with using non-coherent detection the relation of improvement with increasing amount of SNR and values of M-ary of QAM. The progress of the BER is low with SNR and its almost ignorable when $\mathrm{k}>3$. As a comparison with using results in Fig. 2-b, the improvement is obvious with using coherent detection method. With utilizing fixed values for amplitude of all transmitted samples $(A=\sqrt{0.5})$, amount of improvement in BER with SNR with using coherent detection, see Fig. (3-a). While amount of progress is almost slow with non-coherent scheme, as shown in Fig. (3-b).

It is clear from the achieved results in figures 2 and 3 that for fixed values of $\mathrm{M}$, less power is needed in order to achieve the same BER with utilizing coherent detection method regardless normalize the transmitted power or considering power of symbols individually. 
International Journal of Distributed and Parallel Systems (IJDPS) Vol.2, No.2, March 2011

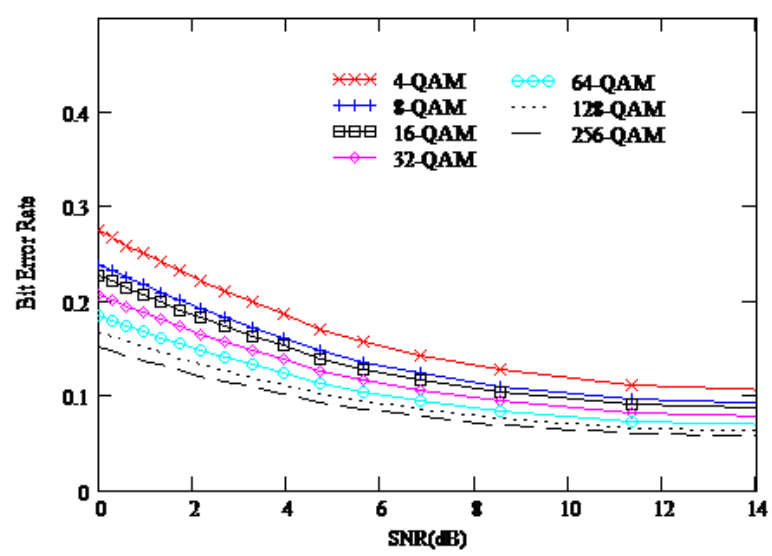

(a)

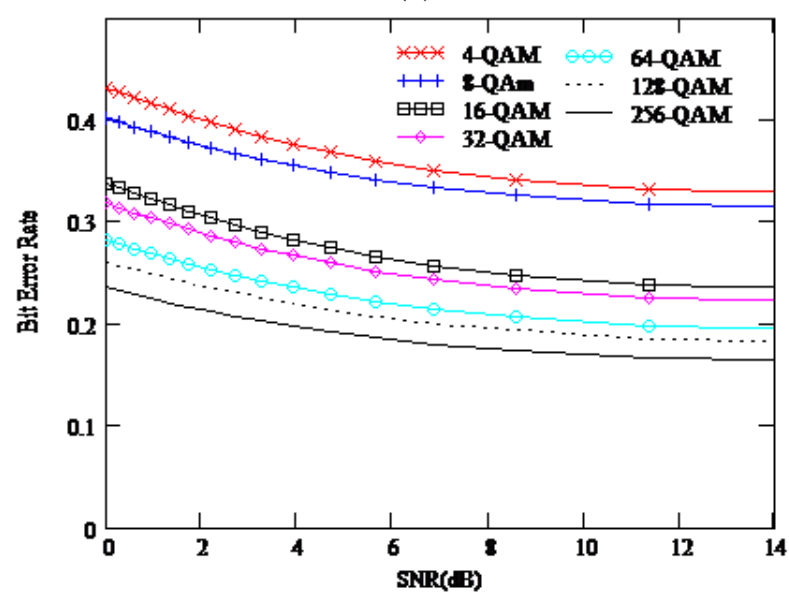

(b)

Figure 3. Bit error rate as a function of SNR for multi levels of QAM: 4, 8, 16, 32, 64, 128, and 256 over the BFWA channel for: (a) coherent and (b) non-coherent detection. (amplitude is constant for all types $\mathrm{A}=(0.5)^{0.5}$ ).

\section{Conclusions}

This paper presents an analytical model for evaluating performance of the M-QAM for BFWA for two detection schemes, namely, coherent and non-coherent. The expressions/relations are derived and investigated for many values of M-ary of QAM: 4, 8, 16, 32, 64, 128, and 256 to evaluate conditional probability of error, bit error probability and average of the probability of error. The derived expressions/relations are analyzed and the achieved results indicate that to achieve a specific value of the probability of error amount of SNR with using coherent detection method is lower than that with using non-coherent one. Also, amount of degradation in the probability of errors with using coherent detection is faster than that with utilizing non-coherent one for all modulation levels in the QAM when the SNR get lower levels. 


\section{Appendix (A)}

\section{4-QAM}

For non-coherent detection scheme

The conditional bit error probability $P_{a}$ is computed as:

$$
\begin{gathered}
P_{a}=\frac{1}{2} \gamma_{a} \\
=\frac{1}{2}\left[P_{1 I}+P_{1 Q}+2 . P_{2}\right]
\end{gathered}
$$

In this case, the error probability can be computed as:

$P_{b e r}=\frac{1}{2}\left[Q\left(Z_{1}\right)+Q\left(Z_{2}\right)\right]+Q\left(Z_{1}\right) Q\left(Z_{2}\right)$

The average probability of error is derived to be as:

$\bar{P}_{b e r}=\frac{1}{4}+Q(\psi)$

\section{8-QAM}

The conditional bit error probabilities: $P_{a}$ and $P_{b}$ are computed as:

$$
\begin{aligned}
& P_{a}=\frac{1}{3} \gamma_{a}=\frac{1}{3}\left[2 . P_{1 I}+P_{1 Q}+4 . P_{2}\right] \\
& P_{b}=\frac{1}{3} \gamma_{b}=\frac{1}{3}\left[P_{1 I}+P_{1 Q}+2 . P_{2}\right]
\end{aligned}
$$

In this case, the error probability can be computed as:

$$
\begin{gathered}
P_{\text {ber }}=\frac{1}{8} \cdot \frac{4}{3}\left[2 \cdot P_{1 I}+P_{1 Q}+4 \cdot P_{2}+P_{1 I}+P_{1 Q}+2 \cdot P_{2}\right] \\
\quad=\frac{1}{6}\left[3 \cdot Q\left(Z_{1}\right)+2 \cdot Q\left(Z_{2}\right)+6 \cdot Q\left(Z_{1}\right) Q\left(Z_{2}\right)\right]
\end{gathered}
$$

Hence, after some manipulation steps of math, the average of the probability is:

$\bar{P}_{b e r}=\frac{1}{4}+\frac{5}{6} \cdot Q(\psi)$

\section{6-QAM [3]:}

The conditional bit error probabilities: $P_{a}, P_{b}$, and $P_{c}$ are computed as:

$$
\begin{aligned}
& P_{a}=\frac{1}{4} \gamma_{a}=\frac{1}{4}\left[2 . P_{1 I}+2 . P_{1 Q}+8 . P_{2}\right] \\
& P_{b}=\frac{1}{4} \gamma_{b}=\frac{1}{4}\left[P_{1 I}+2 . P_{1 Q}+4 . P_{2}\right] \\
& P_{b}=\frac{1}{4} \gamma_{c}=\frac{1}{4}\left[P_{1 I}+P_{1 Q}+2 . P_{2}\right]
\end{aligned}
$$

While the probability of the bit error rate is:

$P_{\text {ber }}=\frac{5}{16} Q\left(Z_{1}\right)+\frac{7}{16} Q\left(Z_{2}\right)+\frac{18}{16} Q\left(Z_{1}\right) Q\left(Z_{2}\right)$

Then, the average of the error probability is:

$\bar{P}_{b e r}=\frac{5}{32}+Q(\psi)$

\section{2-QAM}

The conditional bit error probabilities $P_{a}, P_{b}, P_{c}, P_{d}$ and $P_{e}$ are computed as:

$$
\begin{aligned}
& P_{a}=P_{b}=\frac{1}{5}\left[2 . P_{1 I}+2 . P_{1 Q}+8 . P_{2}\right] \\
& P_{c}=\frac{1}{5}\left[2 . P_{1 I}+2 . P_{1 Q}+6 . P_{2}\right] \\
& P_{d}=\frac{1}{5}\left[P_{1 I}+2 . P_{1 Q}+4 . P_{2}\right]
\end{aligned}
$$




$$
P_{e}=\frac{1}{5}\left[P_{1 I}+P_{1 Q}+4 . P_{2}\right]
$$

After some manipulation steps, we can formulate probability of bit error rate as:

$P_{\text {ber }}=\frac{6}{20} Q\left(Z_{1}\right)+\frac{7}{20} Q\left(Z_{2}\right)+\frac{23}{20} \cdot Q\left(Z_{1}\right) Q\left(Z_{2}\right)$

The average probability of error is:

$\bar{P}_{b e r}=\frac{6}{40}+\frac{37}{40} \cdot Q(\psi)$

\section{4-QAM}

The conditional bit error probabilities: $P_{a}, P_{b}, P_{c}, P_{d}, P_{e}, P_{j}, P_{g}, P_{h}, P_{i}$ and $P_{j}$ are computed as:

$$
\begin{aligned}
& P_{a}=P_{b}=P_{c}=P_{d}=P_{e}=P_{f}=\frac{1}{6}\left[2 . P_{1 I}+2 . P_{1 Q}+8 . P_{2}\right] \\
& P_{g}=P_{h}=P_{i}=\frac{1}{6}\left[P_{1 I}+2 . P_{1 Q}+4 . P_{2}\right] \\
& P_{j}=\frac{1}{6}\left[P_{1 I}+P_{1 Q}+2 . P_{2}\right]
\end{aligned}
$$

In this case, the probability of bit error rate is:

$$
P_{\text {ber }}=\frac{1}{6 \times 64}\left[100 \cdot Q\left(Z_{1}\right)+124 \cdot Q\left(Z_{2}\right)+392 \cdot Q\left(Z_{1}\right) Q\left(Z_{2}\right)\right]
$$

Then, we can obtain the average of the error probability as:

$\bar{P}_{\text {ber }}=\frac{50}{384}+\frac{320}{384} \cdot Q(\psi)$

\section{8-QAM}

The conditional bit error probabilities: $P_{a}, P_{b}, P_{c}, P_{d}, P_{e}, P_{i}, P_{f}, P_{g}, P_{h} P_{j}, P_{k}, P_{n}, P_{o}, P_{p}$

, $P_{q}$ and $P_{r}$ are computed as:

$$
\begin{aligned}
P_{a}=P_{b}=P_{c}=P_{d}=P_{e}=P_{i}=P_{f}=P_{g}=P_{h}=P_{k}=P_{l}=P_{m} \\
=\frac{1}{7}\left[n_{a 1 I} P_{1 I}+n_{a 1 Q} \cdot P_{1 Q}+n_{a 2} \cdot 2 \cdot P_{2}\right] \\
=\frac{1}{7}\left[2 \cdot P_{1 I}+2 \cdot P_{1 Q}+8 \cdot P_{2}\right] \\
P_{j}=\frac{1}{7}\left[2 \cdot P_{1 I}+2 \cdot P_{1 Q}+6 \cdot P_{2}\right] \\
P_{n}=\frac{1}{7}\left[2 . P_{1 I}+P_{1 Q}+6 \cdot P_{2}\right] \\
P_{o}=P_{p}=P_{q}=\frac{1}{7}\left[P_{1 I}+2 \cdot P_{1 Q}+4 \cdot P_{2}\right] \\
P_{r}=\frac{1}{7}\left[P_{1 I}+P_{1 Q}+2 \cdot P_{2}\right]
\end{aligned}
$$

The probability of bit error rate is:

$P_{b e r}=\frac{1}{128}\left[n_{a} P_{a}+n_{b} \cdot P_{b}+\cdots+n_{r} \cdot P_{r}\right]$

With performing some manipulation steps, we get:

$P_{\text {ber }}=\frac{1}{4} Q\left(Z_{1}\right)+\frac{15}{56} Q\left(Z_{2}\right)+\frac{107}{112} Q\left(Z_{1}\right) Q\left(Z_{2}\right)$

The average of the error probability can be achieved as:

$\bar{P}_{\text {ber }}=\frac{1}{8}+\frac{167}{224} \cdot Q(\psi)$ 


\section{6-QAM}

$P_{a}=\frac{1}{8}\left[n_{a 1 I} P_{1 I}+n_{a 1 Q} \cdot P_{1 Q}+n_{a 2} \cdot 2 \cdot P_{2}\right]$
$=\frac{1}{8}\left[2 \cdot P_{1 I}+2 \cdot P_{1 Q}+8 \cdot P_{2}\right]$

From the space diagram with some further manipulations we noted that:

$P_{a}=P_{b}=P_{c}=P_{d}=P_{e}=P_{f}=P_{g}=P_{h}=P_{i}=P_{j}=P_{k}=P_{l}=P_{m}=P_{n}=P_{o}=$

$P_{p}=P_{q}=P_{r}=P_{s}=P_{t}=P_{u}=P_{x 1}=P_{x 2}=P_{x 3}=P_{x 4}=P_{x 5}=P_{x 6}=P_{x 7}$

$P_{y 2}=\frac{1}{8}\left[P_{1 I}+2 . P_{1 Q}+4 . P_{2}\right]$

$=P_{y 3}=P_{y 4}=P_{y 5}=P_{y 6}=P_{y 7}=P_{y 8}$

$P_{y 1}=\frac{1}{8}\left[P_{1 I}+P_{1 Q}+2 . P_{2}\right]$

While the probability error rate is:

$$
\begin{aligned}
P_{b e r}=\frac{1}{8 \times 256}\left[(7.4+21 \times 8)\left(2 . P_{1 I}+2 . P_{1 Q}+8 . P_{2}\right)\right. \\
\left.+(7 \times 8)\left(P_{1 I}+2 . P_{1 Q}+4 . P_{2}\right)+4 .\left(P_{1 I}+P_{1 Q}+2 . P_{2}\right)\right]
\end{aligned}
$$

With performing some manipulation steps, we achieve the following expression for the probability error rate:

$P_{\text {ber }}=\frac{1}{512}\left[113 \cdot Q\left(Z_{1}\right)+127 \cdot Q\left(Z_{2}\right)+450 \cdot Q\left(Z_{1}\right) Q\left(Z_{2}\right)\right]$

Therefore, the average of the probability of error becomes as:

$\bar{P}_{\text {ber }}=\frac{113}{1024}+\frac{352}{512} \cdot Q(\psi)$

\section{Appendix (B)}

For coherent detection scheme

Let $Y=\frac{A\left|h_{o}\right|^{2}}{\sqrt{N_{W / 2}}}, P_{1}=Q(Y), P_{2}=Q^{2}(Y)$

\section{4-QAM}

$P_{a}=\frac{1}{k}\left(n_{a 1} \cdot 1 \cdot P_{1}+n_{a 2} \cdot 2 \cdot P_{2}\right)=\frac{1}{2}\left(2 \cdot P_{1}+2 \cdot P_{2}\right)=P_{1}+P_{2}$

Then, the probability of error can be achieved as:

$P_{\text {ber }}=Q(Y)+Q^{2}(Y)$

\section{8-QAM}

$P_{a}=\frac{1}{3}\left(3 \cdot P_{1}+4 \cdot P_{2}\right), P_{b}=\frac{1}{3}\left(2 \cdot P_{1}+2 \cdot P_{2}\right)$

So, the probability of error obtained as:

$P_{b e r}=\frac{5}{6} Q(Y)+Q^{2}(Y)$

\section{6-QAM}

$P_{a}=\frac{1}{4}\left(4 \cdot P_{1}+8 \cdot P_{2}\right), P_{b}=\frac{1}{4}\left(3 \cdot P_{1}+4 \cdot P_{2}\right)$, and $P_{c}=\frac{1}{4}\left(2 \cdot P_{1}+2 \cdot P_{2}\right)$

So,

$P_{\text {ber }}=\frac{3}{4} Q(Y)+\frac{9}{8} Q^{2}(Y)$ 


\section{2-QAM}

$P_{a}=P_{b}=\frac{1}{5}\left(4 \cdot P_{1}+8 \cdot P_{2}\right), P_{c}=\frac{1}{5}\left(4 \cdot P_{1}+6 \cdot P_{2}\right)$

$P_{d}=\frac{1}{5}\left(3 . P_{1}+4 \cdot P_{2}\right), P_{e}=\frac{1}{5}\left(2 \cdot P_{1}+4 \cdot P_{2}\right)$

The bit error probability is thus

$P_{\text {ber }}=\frac{13}{20} Q(Y)+\frac{23}{20} Q^{2}(Y)$

\section{4-QAM}

$P_{a}=P_{b}=P_{c}=P_{d}=P_{e}=P_{f}=\frac{1}{6}\left(4 \cdot P_{1}+8 . P_{2}\right)$

$P_{g}=P_{h}=P_{i}=\frac{1}{6}\left(3 . P_{1}+4 \cdot P_{2}\right)$

$P_{j}=\frac{1}{6}\left(2 \cdot P_{1}+2 \cdot P_{2}\right)$

Therefore, $P_{b e r}=\frac{14}{24} Q(Y)+\frac{49}{48} Q^{2}(Y)$

\section{8-QAM}

$P_{a}=P_{b}=P_{c}=P_{d}=P_{e}=P_{i}=P_{f}=P_{g}=P_{h}=P_{k}=P_{l}=P_{m}=\frac{1}{7}\left(4 . P_{1}+8 . P_{2}\right)$

$P_{j}=\frac{1}{7}\left(4 \cdot P_{1}+6 \cdot P_{2}\right)$

$P_{n}=\frac{1}{7}\left(3 \cdot P_{1}+6 \cdot P_{2}\right)$

$P_{o}=P_{p}=P_{q}=\frac{1}{7}\left(3 \cdot P_{1}+4 \cdot P_{2}\right)$

$P_{r}=\frac{1}{7}\left(2 . P_{1}+2 . P_{2}\right)$

The bit error probability is thus

$P_{b e r}=\frac{58}{112} Q(Y)+\frac{107}{112} Q^{2}(Y)$

\section{6-QAM}

From the space diagram with some further manipulations we noted that:

$$
\begin{aligned}
& P_{a}=P_{b}=P_{c}=P_{d}=P_{e}=P_{f}=P_{g}=P_{h}=P_{i}=P_{j}=P_{k}=P_{l}=P_{m} \\
& =P_{n}=P_{o}=P_{p}=P_{q}=P_{r}=P_{s}=P_{t}=P_{u}=P_{x 1}=P_{x 2}=P_{x 3}=P_{x 4}=P_{x 5}=P_{x 6} \\
& =P_{x 7}=\frac{1}{8}\left[4 \cdot P_{1}+8 \cdot P_{2}\right] \\
& P_{y 2}=\frac{1}{8}\left[3 \cdot P_{1}+4 . P_{2}\right] \\
& =P_{y 3}=P_{y 4}=P_{y 5}=P_{y 6}=P_{y 7}=P_{y 8} \\
& P_{y 1}=\frac{1}{8}\left[2 . P_{1}+2 . P_{2}\right]
\end{aligned}
$$

While the probability error rate is:

$$
\begin{gathered}
P_{\text {ber }}=\frac{1}{8 \times 256}\left[(7 \times 4)\left(4 . P_{1}+8 . P_{2}\right)+(21 \times 8)\left(4 . P_{1}+8 . P_{2}\right)+7 \times 8\left(3 . P_{1}\right.\right. \\
\left.\left.+4 . P_{2}\right)+7\left(2 . P_{1}+2 . P_{2}\right)\right]
\end{gathered}
$$


After doing some manipulations yields:

$P_{b e r}=\frac{120}{256} Q(Y)+\frac{225}{256} Q^{2}(Y)$

\section{REFERENCES}

[1] Arjuna Sathiaseelan \& Gorry Fairhurst, (2007) "Multimedia congestion control for broadband wireless networks", Mobile and Wireless Communications Summit, 16th IST, pp.1-5.

[2] William Webb, (2001) "Broadband fixed wireless access as a key component of the future integrated communications environment", IEEE Commun. Mag., Vol. 39, No. 9, pp. 115- 121.

[3] Pei Xiao \& Rolando Carrasco, (2005) "Performance analysis of conventional detection in BFWA systems", Second IFIP international conference on Wireless and optical communications networks, WOCN, pp. 447-452.

[4] Isabelle Tardy \& Ole Grøndalen, (2005) "On the Role of Future High-Frequency BFWA Systems in Broadband Communication Networks", IEEE Communications Magazine, Vol. 43, No. 2, pp. 138144.

[5] IEEE Standard, (2003) "Part 16: air interface for fixed broadband wireless access systems amendment 2: media access control modifications and additional physical layer specifications for 2$11 \mathrm{GHz} "$, Standard IEEE 802.16a.

[6] Stuber, G.L. Barry, J.R., McLaughlin, S.W. et al., (2004) "Broadband MIMO-OFDM wireless communications", Proc., 92, pp. 271-294.

[7] Masataka Nakazawa, (2008) "Challenges to FDM-QAM Coherent Transmission with Ultrahigh Spectral Efficiency", ECOC, Brussels, Belgium, Vol. 2, pp. 45-48.

[8] Kazuki Maeda \& Shozo Komaki, (2000) "Error Statistics of 64-QAM Signal in AM/64-QAM Hybrid Optical Transmission", J. Lightwave Technol., Vol. 18, No. 10, pp. 1348-1354.

[9] Ivan B. Djordjevic \& Bane Vasic, (2006) "Multilevel coding in M-ary DPSK/Differential QAM high-speed optical transmission with direct detection", J. of Lightwave technology, Vol. 24, No. 1, pp. 420-428.

[10] M. S. Patterh, T. S. Kamal and B. S. Sohi , (2000) "Performance of coherent square M-QAM in wireless frequency non-selective slowly fading channels", IEEE. International Conference on Communication Technology Proceedings, WCC - ICCT., Vol.2, pp. 1358 - 1360.

[11] Toriyama, Y.; Kojima, K.; Taniguchi, T.; Miao Zhang; Hirokawa, J., (2010) " Multi-level QAM single-carrier high-efficiency broadband wireless system for millimeter-wave applications", IEEE Radio and Wireless Symposium (RWS), pp. 677 - 680.

[12] Beeta Tarokh \& Hamid R. Sadjadpour, (2003) "Construction of OFDM M-QAM sequences with low peak-to-average power ratio", IEEE Trans. on Comm. Vol. 51, No. 1, pp. 25-28.

[13] Bernard Sklar, (2005) Digital Communications Fundamentals and Applications, 2nd ed. New Jersey, Prentice Hall.

[14] Leszek Szczecinski, Cristian González, and Sonia Aïssa, (2006) "Exact expression for the BER of rectangular QAM with arbitrary constellation mapping", IEEE Trans. On Commun., Vol. 54, No. 3. pp. 389-392.

[15] Zafar Q. Taha and Xian Li, (2007) "Low PMEPR code based on STAR-16-QAM constellation for OFDM", IEEE Commun. letters, Vol. 11, No. 9, pp. 747 - 749.

[16] Xian Liu and Hsiao-Chuan Wu, (2010) "Novel asterisk 16QAM constellation for COFDM", IEEE Comm. letters, Vol. 14, No. 7, pp. $596-598$.

[17] M. K. Khan, R. A. Carrasco, I. J. Wassell and J. A. Neasham, (2008) "Performance comparison of low density parity check codes using square root kalman equalization and orthogonal frequency division multiplexing techniques for broadband fixed wireless access systems", IET Commun., Vol. 2, No. 2, pp. 272-283. 


\section{Authors}

Haider M. AlSabbagh (haidermaw@ieee.org) received his $\mathrm{Ph} . \mathrm{D}$. degree from school of electronic information and electrical engineering (SEIEE), Shanghai Jiao Tong University in 2008, and his M.S. degree in communications and electronics engineering from Basrah University in 1996. From 1996 to 2002, he worked in Basrah University as a lecturer. Currently, he is an associate professor in Basra University. His research interests include wireless communication, mobile and wireless networks, data communications, information networks, optical communications, routing. Dr. Haider is a member of editorial board for several journals and occupies a committee member of many international conferences, also he has been serving as a referee for many international journals: IETcommunications, IJIPM, IJACT, and conferences, such as: IEEE WCNC 2010 Networks, ICCAIE 2010, EPC-IQ01, MICCCA2009, IET-WSN 2010. Dr. Haider is a member of IEEE.

Ali Amin (ali_amin@ieee.org) has got his MSc in electrical engineering in 2001, from Basra University, where he has been working as a lecturer for wireless and microwave communications engineering. His interesting research areas include: wireless communications, microwave engineering, and mobile communications. Mr. Ali is a member of IEEE.
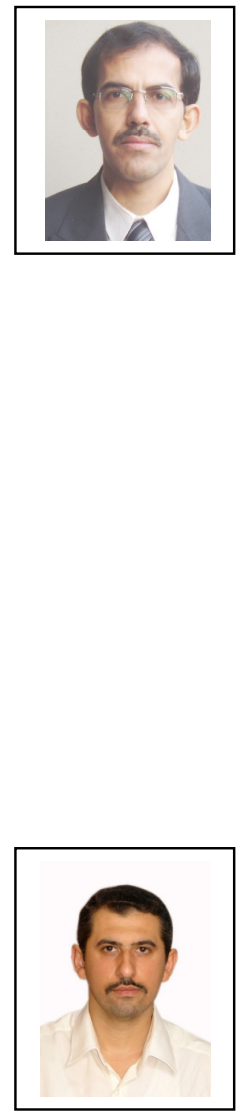